\title{
The Improvement on High Voltage Power Supply Control System of
}

\section{Electrostatic Precipitators}

\author{
Li Yan-jie ${ }^{1, a}$, Chen Yu-xiang ${ }^{2, b}$ \\ 1,2University of Science and Technology LiaoNing, China

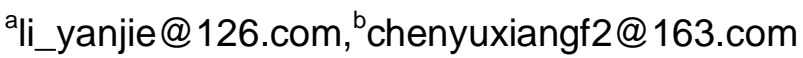

\begin{abstract}
Key words: high voltage power supply; the best spark rate; automatic control system; rectifying equipment
\end{abstract}

Abstract. This paper expounds the control system structure, working principle and characteristics of high voltage power supply of electrostatic precipitator, introducing the main technical parameters and performance of high voltage power supply control system of the new type electrostatic precipitator, and finally analysing the common faults in the operation of equipment and puts forward the method to solve the fault. Operation results show that the system is stable and reliable, and overall performance and the efficiency of dust control have been improved significantly. The scheme has been well applied in the field of environmental protection and dust removal.

\section{Introduction}

With the continuous industrial development in our country, more and more attention has been drawn towards the protection of environment. Especially because of the discharge of PM2.5, there are more stringent requirements for the discharge of pollutants. So it is imperative to reform of electrostatic precipitators. Traditional electrostatic precipitator rectifier equipment control system often results in multiple faults in operation which contribute to the instability of rectifier devices' working condition, such as: slow speed of sparks tracking, unable to take charge measurement and the display of static parameters, low control accuracy etc. Aiming at the existing defects of equipment, electrostatic precipitators of high voltage power supply system has been remodified by using the microcomputer control of high voltage rectifier equipment. Until now, electrostatic precipitator is in good wording order and the dust removal effect has been improved obviously.

\section{Main structure and working principle}

Main structure .In the electric dust catcher system, electrical system is mainly composed of high voltage silicon rectifier control cabinet, rectifier transformer and reactor. Digital control is adopted in the system. The structure of the control system is shown in figure 1, core is digital signal processor. The components of the system exchange information with each other through a digital signal processor[1]. The control of the silicon rectifier transformer dc output voltage is realized through mutual information exchange, communication and data processing process between each part and digital signal processor. 


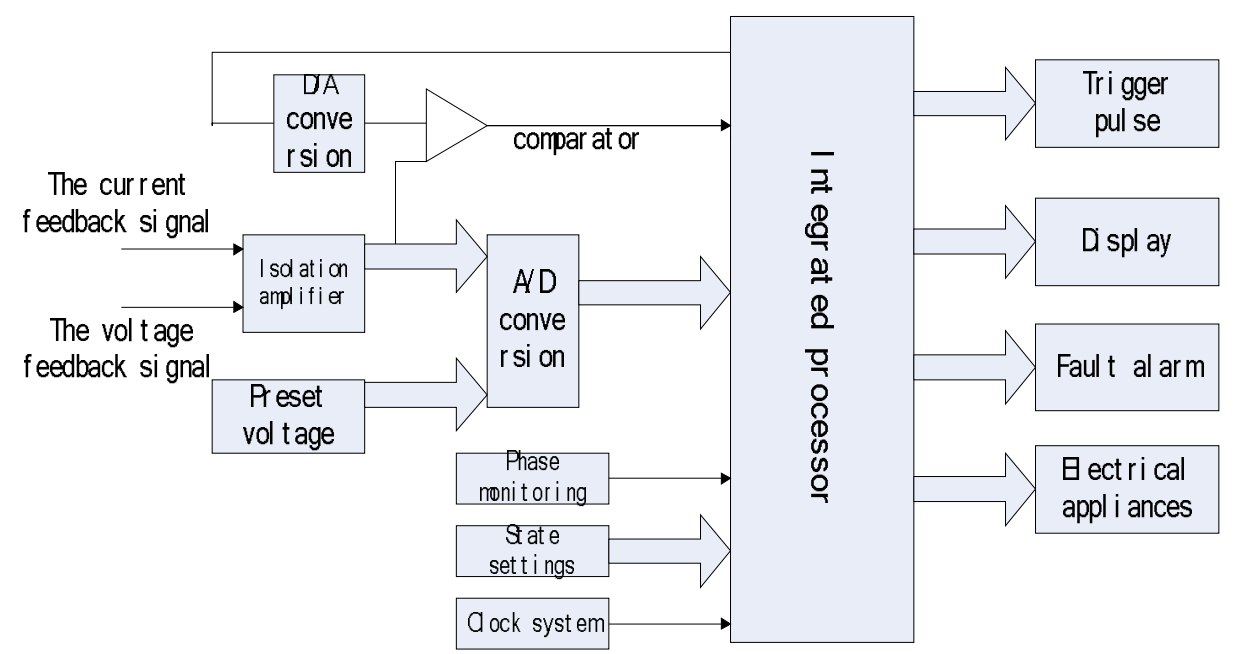

Fig.1 Control system structure

Working principle. High voltage power supply equipment to provide electricity to oneself of electrostatic precipitators, producing corona discharge between the corona electrode and dust collector and letting the charged dust particles driven to dust collector. In order to make the electrostatic precipitators reach the highest dust removal efficiency in different specifications and different conditions, the power of the electric dust catcher must also have a set of functional control system. High voltage power supply equipment electrostatic precipitators mainly includes: voltage step-up transformer that can increase the line voltage; rectifier transforming the high voltage alternating current into the high voltage direct current; high voltage power supply control cabinet. By the access of single-phase electric $380 \mathrm{VAC}$ and the start of main circuit of ac contactor, control system works. First, the controller reads out the key parameters that are saved at the original setting value and the last stop, which are used as the basis of adjusting conduction angle of silicon controlled rectifier[2]. At the same time the sampling voltage and current signals are provided to the $\mathrm{A} / \mathrm{D}$ converter. Then A/D sends back these data to the controller after conversion respectively. The controller analyzes those data, identifies the running state of the electric field, and then compares them with the original value to regulate the primary side of main circuit conduction angle of silicon controlled rectifier. Eventually, trigger the step-up transformer rectifier to produce negative pressure to the electric field, then the electric field works by a certain set of control mode. As shown in figure 2.

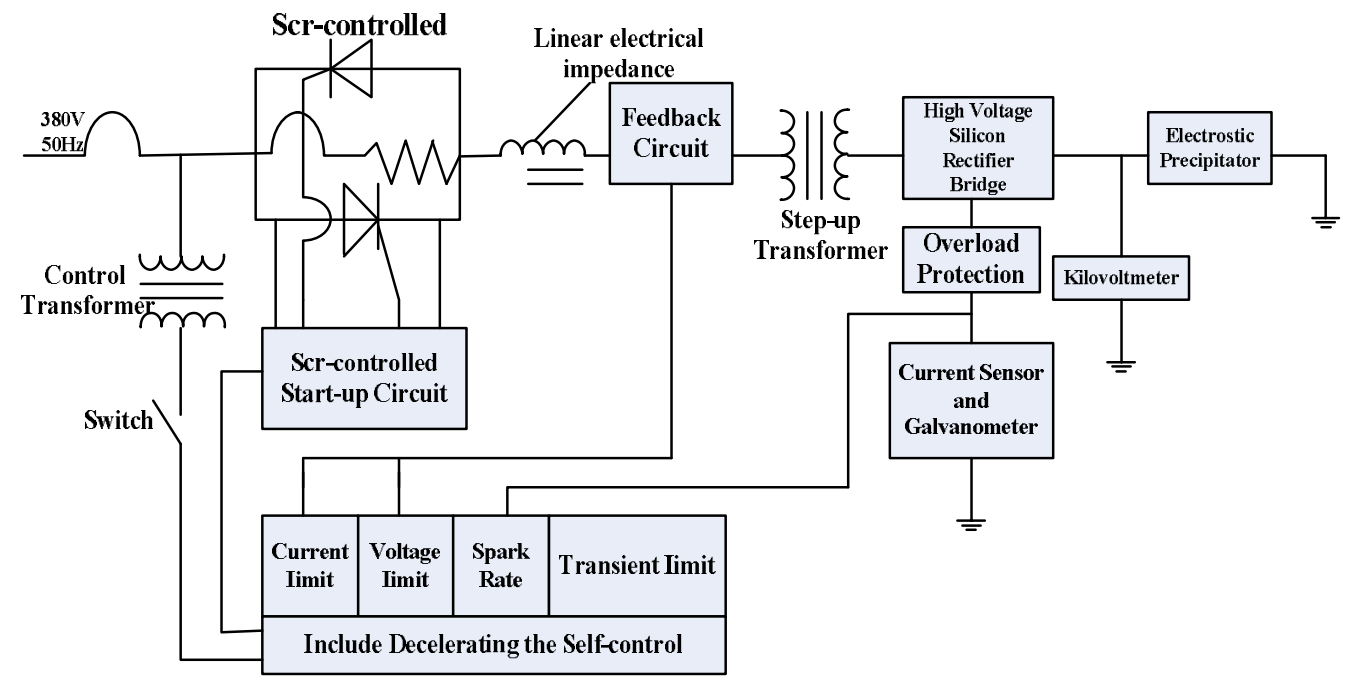

Fig.2 Schematic diagram of major loop of high voltage power supply control system 


\section{Main technical indicators and performance}

Main technical indicators . The purpose of improving the power supply of electrostatic precipitator is to obtain more and more high efficiency of dust removal. Dust collector at work, precipitator power supply voltage should as far as possible to close to the spark discharge voltage and electrostatic precipitator power supply to be able to quickly judge and deal with the spark discharge, and achieve certain spark rate[3]. When electrostatic precipitators work, the working voltage of power supply should be as high as possible to close to spark discharge voltage, and to achieve a certain spark rate. In this paper GGAJ02 high voltage silicon rectifier equipment is classified by the rated output voltage, (secondary voltage) and rated current (secondary current). The secondary rated output voltage is divided into three gears: $60 \mathrm{kv}, 72 \mathrm{kv}, 90 \mathrm{kv}$ three levels. AC input voltage: single-phase $380 \mathrm{v}$; frequency: $50 \mathrm{~Hz}$.All of the electrical operation data and parameters can be displayed on the color touch screen which can not only display the digital information, but can show working mode, such as: spark times per minute, spark occurs, alarm/state, secondary current, etc.

Main technical performance .It has process detection performance and soft start performance. Process detection circuit is used to detect the time interval of spark and arc. If the last programming time interval after the creation of spark and arc does not produce sparks and arc, fast rate of climb function automatically. If no spark or arc has been detected during the programming time, the system will automatically increase current by rising rate preset, which helps avoid the situation of long time with low power which mentioned below. Soft start features can prevent the key components, such as silicon, silicon rectifier transformer, being impacted by the instantaneous current that may cause equipment damage phenomenon.

\section{Analysis and treatment for typical faults .}

Analysis and treatment for typical faults one.Spark discharge phenomenon occurs in electric field of dust collector, but the control system does not detect it; or no spark discharge phenomenon occurs in electric field of dust collector, but the control system detects the spark discharge phenomenon. Such malfunctions are due to the setup problem of spark detection sensitivity. Adjust GGAJ02 - WJB spark sensitivity on internal PCB potentiometer integrated controller. Turn it counter clock wise to adjust for spark control sensitivity enhancement and vice versa.

Analysis and treatment for typical faults two.After operating equipment for a period of time, secondary voltage and secondary current gradually decrease and the dust removal effect becomes weaken. This may be due to the long-term work under a single mode. Steps below can be followed to do adjustment. First, adjust the low voltage control system rapping periodicity set smaller rapping periodicity. Then, increase vibration or change the mode of vibration. Finally, use "step-down shaking" operation mode. After working for a period of time, observe dust removal effect.

\section{Main characteristic of the improved high voltage power supply control system}

Control accuracy and real-time control .The system uses the embedded dual core CPU structure design to ensure the control accuracy and real-time control; data display and keyboard adopt color touch-screen operation that can not only display the various real-time operating mode parameters of the equipment operation and the volt-ampere characteristic curve, but show history running state and alarm data, etc. Examined by password access mechanism, modify and set limit operation parameters through the virtual keyboard for the color touch screen. To ensure the reliability of spark 
(or arc) tracking control, spark detection circuit of hardware and the software arc detection calculation step are independent of each other.

Fault alarm fault handling . The system has a variety of fault alarm processing functions and the products have system short circuit alarm and fault handling; system open alarm and fault handling; slant thyristor excitation alarm and fault handling; transformer oil temperature, gas overrun alarm and fault handling; user defined alarm and fault handling.

Real-time control modes. The system has a variety of real-time control modes: tracking control; peak tracking control; constant current, constant voltage control; pulse power supply control; intermittent power supply control; arc response control; flow cut-off control; step-down vibrating control; flue gas turbidity meter closed-loop control; manual and automatic switch control each other and so on. All the control modes mentioned can response separately and can also combine a variety of control functions to meet the demand of the actual operation condition.

\section{Conclusions}

High voltage power supply control system of the electrostatic precipitators is the key to enhance dust collecting efficiency. Improvement of the high voltage power supply control system adopts the embedded dual core CPU structure design that can be adjusted effective value voltage in time according to the situation of live load so as to achieve the best dust removal efficiency. At the same time, electric precipitator system has greatly improved in aspects of reliability and control performance of the electric dust removal system, network communication, intelligent control and human-machine interface operation, etc. As a result, the improvement of the system will get positive applications.

\section{Acknowledgment}

First and foremost, I would like to show my deepest gratitude to Professor C.L. LING. Conversations with her have been most helpful. I shall extend my thanks to Mr. Ao for all his kindness and help. This work has been supported by The Applied school of University of Science and Technology Liaoning.

\section{References:}

[1]Zhiguang Hu,etc, Electrostatic Precipitator Power Supply Control Technology[J].North China Electric Power University, 1998.

[2]Yu Liu, The Research of the Control System of High Voltage Power Supply Electrostatic Precipitators, Liaoning University of Science and Technology[D]2008.3:18 19.

[3]Jun Chen, Digital Microcomputer Control High Voltage Electrostatic Dedusting Rectifier Equipment Applications[J].Shandong Metallurgical, 1999. 